\title{
DINAMINIO METODO LYGTYS, KAI PALYDOVO ORBITA ARTIMA PARABOLEI
}

\author{
Petras Petroškevičius \\ Geodezijos ir kadastro katedra, Geodezijos institutas, Vilniaus Gedimino technikos universitetas, \\ Saulètekio al. 11, LT-10223 Vilnius-40, Lietuva, el.paštas: petras.petroskevicius@ap.vtu.lt
}

Iteikta 20040120 , priimta 20040210

\begin{abstract}
Santrauka. Gautos kosminès geodezijos dinaminio metodo lygtys. Taikant šias lygtis, pagal išmatuotas palydovo topocentrines koordinates galima patikslinti geopotencialo zoninius ir nezoninius koeficientus, punktu geocentrines koordinates bei palydovo orbitos elementus. Lygtyse vietoje laiko taikomas kintamasis $\sigma_{0}$, siejamas su parabolinio judejjimo, kurio perigejjaus nuotolis tas pats kaip ir palydovo judejjimo, tikraja anomalija $v_{0}, \sigma_{0}=\operatorname{tg} \frac{v_{0}}{2}$. Lygčių koeficientai išreikšti dydžio $\gamma=\frac{1-e}{2}$ laipsninèmis eilutėmis. Čia $e-$ orbitos ekscentricitetas. Eilutès absoliučiai konverguoja esant įvairioms palydovo orbitos ekscentriciteto reikšmėms nuo 0 iki 1. Eilutes galima taikyti, kai $\left|\sigma_{0} \sqrt{\gamma}\right|<1$. Jos ypač efektyvios, kai palydovo orbitos ekscentricitetas artimas vienetui.
\end{abstract}

Raktažodžiai: kosminès geodezijos dinaminis metodas, geopotencialo parametrų nustatymas, beveik parabolinis DŽP judejjimas.

\section{Ivadas}

Kosminès geodezijos dinaminiu metodu nustatomos punktų geocentrinès koordinatès, patikslinami palydovų orbitos elementai bei geopotencialo parametrai. Šiuo metodu itvirtinamos geocentrinès koordinačių sistemos, sudaromi atraminiai didelio tikslumo geodeziniai tinklai. Tokiu būdu sukuriamas vieningas geodezinis pagrindas Žemès formai nustatyti ir jos kitimams tyrinèti [1-3].

Ypač vertingos kosminès geodezijos dinaminio metodo galimybès tiriant Žemès gravitacijos lauką. Remiantis ivairiais DŽP stebejjimo duomenimis ir atliekant palydovų orbitų analizę, patikimai nustatomi žemesniosios eilès zoniniai ir nezoniniai geopotencialo koeficientai, apibūdinantys Žemès formos ir jos gravitacijos lauko bendresnes detales [4-6]. Detalieji Žemès gravitacijos lauko tyrimai atliekami remiantis Žemès paviršiuje atliekamų gravimetrinių matavimų duomenimis [7-8]. Pagal ivvairiais matavimų metodais gautus duomenis sudaromi geopotencialo modeliai [910]. Siekiant išplèsti kosminès geodezijos dinaminio metodo galimybes didinant nustatomų geopotencialo parametrų skaičių ir tikslumą, naudojami žemiau judantys, tam tikros specialios konstrukcijos ir mažiau atmosferos poveikio stabdomi palydovai. Tam tikslui taikomos ir doplerinés sistemos ,palydovas - palydovas“. Tačiau kosminès geodezijos dinaminio metodo galimybès taip pat dideja naudojant palydovus, judančius ịvairiomis orbitomis [4, 11]. Dabar Žemès gravitacijos laukui tirti dažniausiai naudojami palydovai, judantys orbitomis, kurių ekscentricitetas neviršija Laplaso ribos. Tokių palydovu judejjimo teorijose taikomos klasikinès dangaus mechanikos koordinačių eilutès, absoliučiai konverguojančios iki Laplaso ribos [12].

Siekiant išplèsti kosminès geodezijos dinaminio metodo galimybes, tikslinga naudoti palydovus, judančius ištęstomis orbitomis, kurių ekscentricitetas viršija Laplaso ribą [11]. Tokių palydovų judejimo teorijai tinka koordinačių eilutès $\gamma=\frac{1-e}{2}$ dydžio laipsniais [13]. Čia $e$ - orbitos ekscentricitetas. Didejant ekscentricitetui, eilučių konvergavimo greitis didejja. Šiuo atveju judejjimo teorijoje vietoje laiko taikomas naujas kintamasis $\sigma_{0}$, siejamas su parabolinio judejimo, kurio perigèjaus nuotolis tas pats kaip ir palydovo judèjimo, tikraja anomalija $v_{0}, \sigma_{0}=\operatorname{tg} \frac{v_{0}}{2}$. Judejjimo teorija, taikant minètąsias eilutes, tinka, kai $\left|\sigma_{0} \sqrt{\gamma}\right|<1$.

Straipsnyje [14] išnagrinètos orbitinio metodo lygtys, kai palydovo orbita artima parabolei. Šiame straipsnyje pateikiamos kosminès geodezijos dinaminio metodo lygtys, tinkančios palydovams, judantiems beveik parabolinemis orbitomis.

\section{Dinaminio metodo lygtys}

Geopotencialo parametrams, punktų koordinatėms ir palydovo orbitos elementams patikslinti taikysime 
kosminès geodezijos dinamini metodą [4]. Laikysime, kad Žemès paviršiaus punktuose pasaulio laiko momentais $t$ išmatuotos palydovo topocentrinès koordinatès: rektascensija $-\alpha_{0}^{\prime}$, deklinacija $-\delta_{0}^{\prime}$, topocentrinis atstumas $-r_{0}^{\prime}$. Palydovo, judančio beveik paraboline orbita, orbitos elementai: $q$ - perigejaus nuotolis, $\gamma-$ dydis, susijęs su palydovo orbitos ekscentricitetu, $i$ - orbitos plokštumos posvyris $i$ pusiaujo plokštumą, $\Omega$ - kilimo mazgo rektascensija, $\omega$ - perigejjaus kampinis nuotolis nuo kilimo mazgo, $\sigma_{0}$ - dydis, susijęs su parabolinio judejimo tikraja anomalija.

Taikant dinamini metodą patikslinamos punkto stačiakampès koordinatès $X, \quad Y, \quad Z$ Grinvičo koordinačiu sistemoje, pradiniai palydovo orbitos elementai $q_{0}, \gamma_{0}, i_{0}, \Omega_{0}, \omega_{0}, \bar{\sigma}_{0}$ laiko momentu $t_{0}$; geopotencialo ir viršutinès atmosferos parametrai bei Žemès gravitacinis parametras $\mu$.

Parašysime dinaminio metodo lygtis:

topocentrinès rektascensijos -

$$
\begin{aligned}
& \alpha_{o}^{\prime}-\alpha_{c}^{\prime}=\frac{\partial \alpha^{\prime}}{\partial X} \Delta X+\frac{\partial \alpha^{\prime}}{\partial Y} \Delta Y+\frac{\partial \alpha^{\prime}}{\partial Z} \Delta Z+ \\
& +\frac{\partial \alpha^{\prime}}{\partial \Omega_{0}} \Delta \Omega_{0}+\frac{\partial \alpha^{\prime}}{\partial i_{0}} \Delta i_{0}++\frac{\partial \alpha^{\prime}}{\partial \omega_{0}} \Delta \omega_{0}+ \\
& +\frac{\partial \alpha^{\prime}}{\partial q_{0}} \Delta q_{0}+\frac{\partial \alpha^{\prime}}{\partial \gamma_{0}} \Delta \gamma_{0}+\frac{\partial \alpha^{\prime}}{\partial \bar{\sigma}_{0}} \Delta \bar{\sigma}_{0}+ \\
& +\sum_{l=2}^{L} \sum_{m=0}^{l}\left(\frac{\partial \alpha^{\prime}}{\partial C_{l m}} \Delta C_{l m}+\frac{\partial \alpha^{\prime}}{\partial S_{l m}} \Delta S_{l m}\right)+ \\
& +\alpha t+\beta t^{2}+\ldots,
\end{aligned}
$$

topocentrinès deklinacijos -

$$
\begin{aligned}
& \delta_{o}^{\prime}-\delta_{c}^{\prime}=\frac{\partial \delta^{\prime}}{\partial X} \Delta X+\frac{\partial \delta^{\prime}}{\partial Y} \Delta Y+\frac{\partial \delta^{\prime}}{\partial Z} \Delta Z+ \\
& +\frac{\partial \delta^{\prime}}{\partial \Omega_{0}} \Delta \Omega_{0}+\frac{\partial \delta^{\prime}}{\partial i_{0}} \Delta i_{0}+\frac{\partial \delta^{\prime}}{\partial \omega_{0}} \Delta \omega_{0}+ \\
& +\frac{\partial \delta^{\prime}}{\partial q_{0}} \Delta q_{0}+\frac{\partial \delta^{\prime}}{\partial \gamma_{0}} \Delta \gamma_{0}+\frac{\partial \delta^{\prime}}{\partial \bar{\sigma}_{0}} \Delta \bar{\sigma}_{0}+ \\
& +\sum_{l=2}^{L} \sum_{m=0}^{l}\left(\frac{\partial \delta^{\prime}}{\partial C_{l m}} \Delta C_{l m}+\frac{\partial \delta^{\prime}}{\partial S_{l m}} \Delta S_{l m}\right)+ \\
& +\alpha t+\beta t^{2}+\ldots,
\end{aligned}
$$

topocentrinio atstumo iki palydovo -

$$
r_{o}^{\prime}-r_{c}^{\prime}=\frac{\partial r^{\prime}}{\partial X} \Delta X+\frac{\partial r^{\prime}}{\partial Y} \Delta Y+\frac{\partial r^{\prime}}{\partial Z} \Delta Z+
$$

$$
\begin{aligned}
& +\frac{\partial r^{\prime}}{\partial \Omega_{0}} \Delta \Omega_{0}+\frac{\partial r^{\prime}}{\partial i_{0}} \Delta i_{0}+\frac{\partial r^{\prime}}{\partial \omega_{0}} \Delta \omega_{0}+ \\
& +\frac{\partial r^{\prime}}{\partial q_{0}} \Delta q_{0}+\frac{\partial r^{\prime}}{\partial \gamma_{0}} \Delta \gamma_{0}+\frac{\partial r^{\prime}}{\partial \bar{\sigma}_{0}} \Delta \bar{\sigma}_{0}+ \\
& +\sum_{l=2}^{L} \sum_{m=0}^{l}\left(\frac{\partial r^{\prime}}{\partial C_{l m}} \Delta C_{l m}+\frac{\partial r^{\prime}}{\partial S_{l m}} \Delta S_{l m}\right)+ \\
& +\alpha t+\beta t^{2}+\ldots+\frac{\partial r^{\prime}}{\partial \mu} \Delta \mu,
\end{aligned}
$$

čia $\Delta X, \Delta Y, \Delta Z$ - punkto geocentrinių koordinačių pataisos; $\Delta \Omega_{0}, \Delta i_{0}, \Delta \omega_{0}, \Delta q_{0}, \Delta \gamma_{0}, \Delta \bar{\sigma}_{0} \quad-$ pradiniu orbitos elementu pataisos; $C_{l m}, S_{l m}, \Delta C_{l m}, \Delta S_{l m}-$ geopotencialo parametrai ir ju pataisos; $\alpha, \beta, \ldots$ atmosferą apibūdinantys parametrai; $\Delta \mu$ - Žemès gravitacinio parametro pataisa; $\alpha_{c}^{\prime}, \delta_{c}^{\prime}, r_{c}^{\prime} \quad-$ apskaičiuotosios, iskaitant visus trikdymus matavimų momentu, topocentrinių koordinačių reikšmès. Kadangi laiko intervalai nedideli, palydovo orbitos trikdymams nustatyti galima taikyti judejjimo lygčių skaitini integravimą. Gravitaciniams trikdymams nustatyti taip pat galima taikyti sutrikdytojo judejimo formules [15]. Kai lygčių skaičius viršija nežinomujų skaičių, jas galima vertinti kaip pataisų lygtis ir, sudarius normalinių lygčiu sistemą, ieškoti patikimiausių pataisų reikšmių.

Toliau nustatysime dinaminio metodo lygčiu koeficientus, tinkančius, kai DŽP judejjimas beveik parabolinis.

\section{Geopotencialo parametrų pataisų koeficientai}

Šie koeficientai nustatomi pagal formules [4]:

$$
\begin{gathered}
\frac{\partial k}{\partial C_{l m}}=\sum_{s=1}^{6} A_{s} \frac{\partial \theta_{s}}{\partial C_{l m}}, \\
\frac{\partial k}{\partial S_{l m}}=\sum_{s=1}^{6} A_{s} \frac{\partial \theta_{s}}{\partial S_{l m}},
\end{gathered}
$$

čia

$$
A_{s}=\left(\frac{\partial k}{\partial x^{\prime}} \frac{\partial x}{\partial \theta_{s}}+\frac{\partial k}{\partial y^{\prime}} \frac{\partial y}{\partial \theta_{s}}+\frac{\partial k}{\partial z^{\prime}} \frac{\partial z}{\partial \theta_{s}}\right)
$$

$k$ - bet kuri palydovo topocentrinè koordinate, $\theta$ - bet kuris palydovo orbitos sutrikdytas elementas, $x, y, z-$ palydovo geocentrinès inercinès sistemos koordinatès, $x^{\prime}, y^{\prime}, z^{\prime}$ - palydovo topocentrinès koordinatès.

Koeficientams $A_{s}$ nustatyti taikytinos straipsnyje [14] pateiktos formulès. 
Atsižvelgiant i geopotencialo trikdančiosios funkcijos išraišką [15], galima parašyti

$$
\frac{\partial \theta}{\partial C_{l m}}=\frac{\partial \Delta \theta_{l m}^{\prime}}{\partial C_{l m}}=\sum_{p=0}^{l} \sum_{n=0}^{\infty} \frac{\partial \delta \theta_{l m p n}}{\partial C_{l m}} \mid \begin{aligned}
& \sigma_{0} \\
& \bar{\sigma}_{0}
\end{aligned}
$$

$$
\frac{\partial \theta}{\partial S_{l m}}=\frac{\partial \Delta \theta_{l m}^{\prime}}{\partial S_{l m}}=\sum_{p=0}^{l} \sum_{n=0}^{\infty} \frac{\partial \delta \theta_{l m p n}}{\partial S_{l m}} \mid \begin{gathered}
\sigma_{0} \\
\bar{\sigma}_{0}
\end{gathered}
$$

taigi, remdamiesi beveik parabolinès palydovo orbitos elementų pirmosios eilès geopotencialo trikdymus išreiškiančiomis formulèmis [15], gauname

$$
\begin{aligned}
& \frac{\partial \Omega}{\partial C_{l m}}=\sum_{p=0}^{l} \sum_{n=0}^{\infty}\left(\frac{a_{e}}{q}\right)^{l} \frac{\gamma^{n}}{\sqrt{1-\gamma}} \operatorname{cosec} i \frac{\partial F_{l m p}(i)}{\partial i} \times \sum_{v=0}^{\infty}\left\{\begin{array} { l } 
{ N _ { v } ^ { \prime } ( n , - l - 1 , l - 2 p ) } \\
{ N _ { v } }
\end{array} \operatorname { s i n } ^ { \operatorname { c o s } } [ ( l - 2 p ) \omega + m ( \Omega - S ) ] \left[\begin{array}{l}
(2 n+2 v+1)^{-1} \\
(2 n-2 l-2 v-1)^{-1}
\end{array}+\right.\right. \\
& \left.+\begin{array}{l}
(2 n+2 v+3)^{-1} \\
(2 n-2 l-2 v-1)^{-1}
\end{array} \sigma_{0}^{2}\right] \sigma_{0}^{2 n-2 l-1}+{ }_{S_{v}}^{\sigma_{0}^{n+1}}(n,-l-1, l-2 p)^{-\sin } \cos S^{\prime}[(l-2 p) \omega+m(\Omega-S)]\left[\begin{array}{l}
(2 v+2)^{-1} \\
(2 n-2 l-2 v-2)^{-1}
\end{array}+\right. \\
& \left.\left.+\begin{array}{l}
(2 v+4)^{-1} \\
(2 n-2 l-2 v)^{-1}
\end{array} \sigma_{0}^{2}\right] \begin{array}{l}
\sigma_{0}^{2} \\
\sigma_{0}^{2 n-2 l-2}
\end{array}\right\} \sigma_{0}^{ \pm 2 v} \mid \begin{array}{l}
\sigma_{0} \\
\bar{\sigma}_{0}
\end{array},
\end{aligned}
$$

$\frac{\partial \Omega}{\partial S_{l m}}=\sum_{p=0}^{l} \sum_{n=0}^{\infty}\left(\frac{a_{e}}{q}\right)^{l} \frac{\gamma^{n}}{\sqrt{1-\gamma}} \operatorname{cosec} i \frac{\partial F_{l m p}(i)}{\partial i} \sum_{v=0}^{\infty}\left\{\begin{array}{l}N_{v}^{\prime}(n,-l-1, l-2 p) \\ N_{v}\end{array} \begin{array}{c}\sin \\ -\cos \end{array}[(l-2 p) \omega+m(\Omega-S)]\left[\begin{array}{l}(2 n+2 v+1)^{-1} \\ (2 n-2 l-2 v-1)^{-1}\end{array}+\right.\right.$

$\left.+\begin{array}{l}(2 n+2 v+3)^{-1} \\ (2 n-2 l-2 v+1)^{-1}\end{array} \sigma_{0}^{2}\right] \sigma_{0}^{2 n-2 l-1}+{ }_{S_{v}}^{\sigma_{v}^{2 n+1}}(n,-l-1, l-2 p) \sin _{\sin }^{\cos }[(l-2 p) \omega+m(\Omega-S)] \times$

$\left.\left.\times\left[\begin{array}{l}(2 v+2)^{-1} \\ (2 n-2 l-2 v-2)^{-1}\end{array}+\begin{array}{l}(2 v+4)^{-1} \\ (2 n-2 l-2 v)^{-1}\end{array}\right] \sigma_{0}^{2}\right] \begin{array}{l}\sigma_{0}^{2} \\ \sigma_{0}^{2 n-2 l-2}\end{array}\right\} \sigma_{0}^{ \pm 2 v} \mid \begin{aligned} & \sigma_{0} \\ & \bar{\sigma}_{0}\end{aligned}$

$\frac{\partial i}{\partial C_{l m}}=\sum_{p=0}^{l} \sum_{n=0}^{\infty}\left(\frac{a_{e}}{q}\right)^{l} \frac{\gamma^{n}}{\sqrt{1-\gamma}} F_{l m p}(i)[(l-2 p) \operatorname{ctg} i-m \operatorname{cosec} i] \sum_{v=0}^{\infty}\left\{\begin{array}{l}N_{v}^{\prime}(n,-l-1, l-2 p){ }_{\cos }^{-\sin }[(l-2 p) \omega+m(\Omega-S)] \times \\ N_{v}\end{array}\right.$ $\times\left[\begin{array}{l}(2 n+2 v+1)^{-1} \\ (2 n-2 l-2 v-1)^{-1}\end{array}+\begin{array}{l}(2 n+2 v+3)^{-1} \\ (2 n-2 l-2 v+1)^{-1}\end{array} \sigma_{0}^{2}\right] \sigma_{0}^{2 n-2 l-1}-S_{S_{v}}^{\sigma_{v}^{2 n+1}}(n,-l-1, l-2 p)_{\sin }^{\cos }[(l-2 p) \omega+m(\Omega-S)] \times$ $\left.\times\left[\begin{array}{l}(2 v+2)^{-1} \\ (2 n-2 l-2 v-2)^{-1}\end{array}+\begin{array}{l}(2 v+4)^{-1} \\ (2 n-2 l-2 v)^{-1}\end{array}\right] \begin{array}{l}\sigma_{0}^{2} \\ \sigma_{0}^{2 n-2 l-2}\end{array}\right\} \sigma_{0}^{2}+2 v \mid \begin{aligned} & \sigma_{0} \\ & \bar{\sigma}_{0}\end{aligned}$

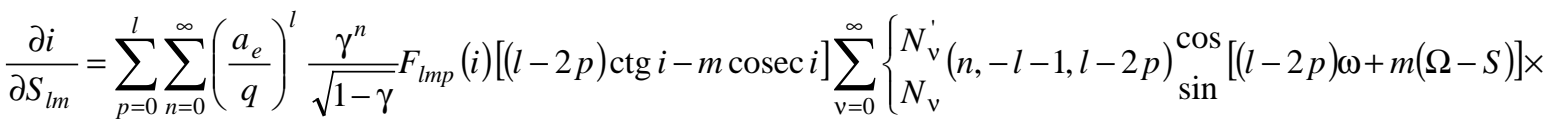

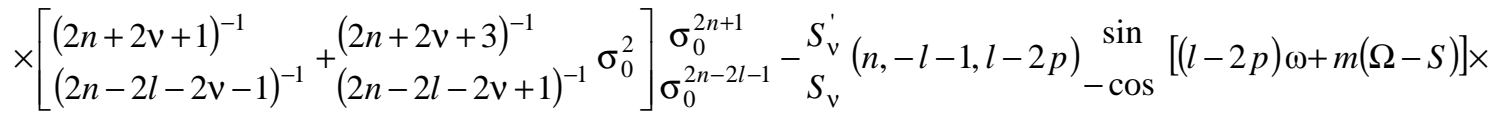

$$
\begin{aligned}
& \left.\times\left[\begin{array}{l}
(2 v+2)^{-1} \\
(2 n-2 l-2 v-2)^{-1}
\end{array}+\begin{array}{l}
(2 v+4)^{-1} \\
(2 n-2 l-2 v)^{-1}
\end{array}\right] \begin{array}{l}
\sigma_{0}^{2} \\
\sigma_{0}^{2 n-2 l-2}
\end{array}\right\} \sigma_{0}^{ \pm 2 v} \mid \begin{array}{l}
\sigma_{0}^{2} \\
\bar{\sigma}_{0}
\end{array}
\end{aligned}
$$

$$
\begin{aligned}
& \frac{\partial \omega}{\partial C_{l m}}=\sum_{p=0}^{l} \sum_{n=0}^{\infty}\left(\frac{a_{e}}{q}\right)^{l} \frac{\gamma^{n} \sqrt{1-\gamma}}{(1-\gamma)} \sum_{v=0}^{\infty}\left\{\begin{array} { l } 
{ N _ { v } ^ { \prime } ( n , - l - 1 , l - 2 p ) } \\
{ N _ { v } }
\end{array} \operatorname { s i n } ^ { \operatorname { c o s } } [ ( l - 2 p ) \omega + m ( \Omega - S ) ] \left[\left(2 F_{l m p}(i)(l-n+1)-\frac{(1-2 \gamma)}{(1-\gamma)} \operatorname{ctg} i \frac{\partial F_{l m p}(i)}{\partial i}\right) \times\right.\right.
\end{aligned}
$$

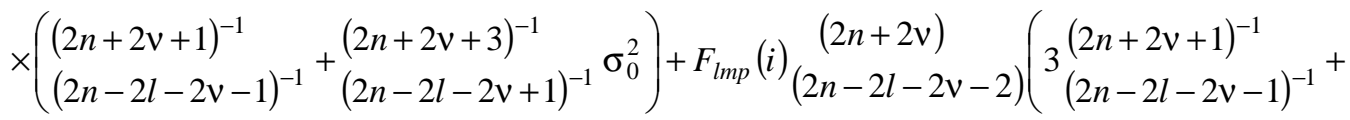

$$
\begin{aligned}
& \left.\left.+\begin{array}{l}
(2 n+2 v+3)^{-1} \\
(2 n-2 l-2 v-1)^{-1}
\end{array} \sigma_{0}^{2}\right)\right]\left[\begin{array}{l}
\sigma_{0}^{2 n+1} \\
\sigma_{0}^{2 n-2 l-1}
\end{array}+{ }_{S_{v}}^{S_{v}^{\prime}}(n,-l-1, l-2 p){ }_{\cos }^{-\sin }[(l-2 p) \omega+m(\Omega-S)] \times\right.
\end{aligned}
$$




$$
\begin{aligned}
& \times\left[\left(2(l-n+1) F_{l m p}(i)-\frac{(1-2 \gamma)}{1-\gamma} \operatorname{ctg} i \frac{\partial F_{l m p}(i)}{\partial i}\right)\left(\begin{array}{l}
(2 v+2)^{-1} \\
(2 n-2 l-2 v-2)^{-1}
\end{array}+\begin{array}{l}
(2 v+4)^{-1} \\
(2 n-2 l-2 v)^{-1}
\end{array} \sigma_{0}^{2}\right)+\right. \\
& \left.\left.+F_{l m p}(i) \begin{array}{l}
(2 v+1) \\
(2 n-2 l-2 v-3)
\end{array}\left(\begin{array}{l}
3 \begin{array}{l}
(2 v+2)^{-1} \\
(2 n-2 l-2 v-2)^{-1}
\end{array} \\
(2 v+4)^{-1} \\
(2 n-2 l-2 v)^{-1}
\end{array} \sigma_{0}^{2}\right)\right] \begin{array}{l}
\sigma_{0}^{2} \\
\sigma_{0}^{2 n-2 l-2}
\end{array}\right\} \sigma_{0}^{ \pm 2 v} \mid \begin{array}{l}
\sigma_{0} \\
\bar{\sigma}_{0}
\end{array},
\end{aligned}
$$

$$
\begin{aligned}
& \frac{\partial \omega}{\partial S_{l m}}=\sum_{p=0}^{l} \sum_{n=0}^{\infty}\left(\frac{a_{e}}{q}\right)^{l} \frac{\gamma^{n} \sqrt{1-\gamma}}{(1-2 \gamma)} \sum_{\mathrm{v}=0}^{\infty}\left\{\begin{array}{l}
N_{\mathrm{v}}^{\prime} \\
N_{\mathrm{v}}
\end{array}(n,-l-1, l-2 p) \underset{-\cos }{\sin }[(l-2 p) \omega+m(\Omega-S)] \times\right. \\
& \times\left[( 2 F _ { l m p } ( i ) ( l - n + 1 ) - \frac { ( 1 - 2 \gamma ) } { ( 1 - \gamma ) } \operatorname { c t g } i \frac { \partial F _ { l m p } ( i ) } { \partial i } ) \left(\begin{array}{l}
(2 n+2 v+1)^{-1} \\
(2 n-2 l-2 v-1)^{-1}
\end{array}+\begin{array}{l}
(2 n+2 v+3)^{-1} \\
(2 n-2 l-2 v+1)^{-1}
\end{array} \sigma_{0}^{2}+F_{l m p}(i) \frac{(2 n+2 v)}{(2 n-2 l-2 v-2)^{2}} \times\right.\right. \\
& \left.\times\left(3 \begin{array}{l}
(2 n+2 v+1)^{-1} \\
(2 n-2 l-2 v-1)^{-1}
\end{array}+\begin{array}{l}
(2 n+2 v+3)^{-1} \\
(2 n-2 l-2 v+1)^{-1}
\end{array} \sigma_{0}^{2}\right)\right] \begin{array}{l}
\sigma_{0}^{2 n+1} \\
\sigma_{0}^{2 n-2 l-1}+{ }_{S_{v}}^{S_{v}^{\prime}}(n,-l-1, l-2 p)
\end{array}{ }_{\sin }^{\cos }[((l-2 p) \omega+m(\Omega-S)] \times \\
& \times\left[\left(2(l-n+1) F_{l m p}(i)-\frac{(1-2 \gamma)}{(1-\gamma)} \operatorname{ctg} i \frac{\partial F_{l m p}(i)}{\partial i}\right)\left(\begin{array}{l}
(2 v+2)^{-1} \\
(2 n-2 l-2 v-2)^{-1}
\end{array}+\begin{array}{l}
(2 v+4)^{-1} \\
(2 n-2 l-2 v)^{-1}
\end{array} \sigma_{0}^{2}\right)+\right. \\
& \left.\left.+F_{l m p}(i) \frac{(2 v+1)}{(2 n-2 l-2 v-3)}\left(\begin{array}{l}
3(2 v+2)^{-1} \\
(2 n-2 l-2 v-2)^{-1}
\end{array}+\begin{array}{l}
(2 v+4)^{-1} \\
(2 n-2 l-2 v)^{-1}
\end{array} \sigma_{0}^{2}\right)\right] \begin{array}{l}
\sigma_{0}^{2} \\
\sigma_{0}^{2 n-2 l-2}
\end{array}\right\} \sigma_{0}^{ \pm 2 v} \mid \begin{array}{l}
\sigma_{0} \\
\bar{\sigma}_{0}
\end{array}
\end{aligned}
$$

$\frac{\partial q}{\partial C_{l m}}=\sum_{p=0}^{l} \sum_{n=0}^{\infty} \frac{a_{e}^{l}}{q^{l-1}} \frac{\gamma^{n}}{(1-2 \gamma)} F_{l m p}(i) \sum_{v=0}^{\infty}\left\{\begin{array}{l}N_{v}^{\prime} \\ N_{v}\end{array}(n,-l-1, l-2 p)\left[2 \sqrt{1-\gamma}(l-2 p)_{\cos }^{-\sin }[(l-2 p) \omega+m(\Omega-S)] \times\right.\right.$

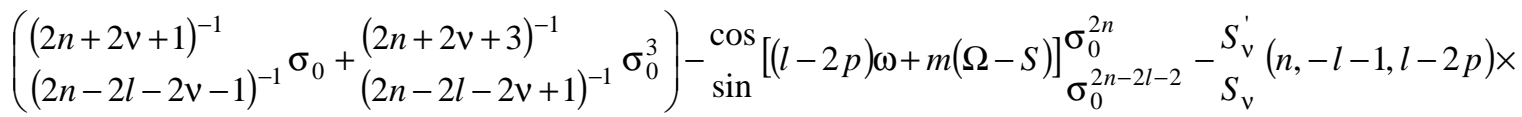

$$
\begin{aligned}
& \times\left[2 \sqrt{1-\gamma}(l-2 p){ }_{\sin }^{\cos }[(l-2 p) \omega+m(\Omega-\Theta)]\left(\begin{array}{l}
(2 v+2)^{-1} \\
(2 n-2 l-2 v-2)^{-1}
\end{array} \sigma_{0}+\begin{array}{l}
(2 v+4)^{-1} \\
(2 n-2 l-2 v)^{-1}
\end{array} \sigma_{0}^{3}\right)+\right.
\end{aligned}
$$

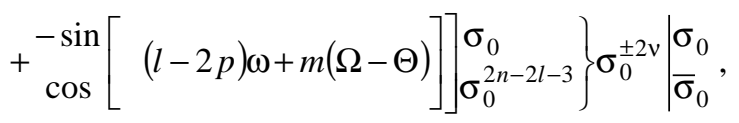

$\frac{\partial q}{\partial S_{l m}}=\sum_{p=0}^{l} \sum_{n=0}^{\infty} \frac{a_{e}^{l}}{q^{l-1}} \frac{\gamma^{n}}{(1-2 \gamma)} F_{l m p}(i) \sum_{\mathrm{v}=0}^{\infty}\left\{\begin{array}{l}N_{\mathrm{v}}^{\prime} \\ N_{\mathrm{v}}\end{array}(n,-l-1, l-2 p)\left[2 \sqrt{1-\gamma}(l-2 p){ }_{\sin }^{\cos }[(l-2 p) \omega+m(\Omega-S)] \times\right.\right.$

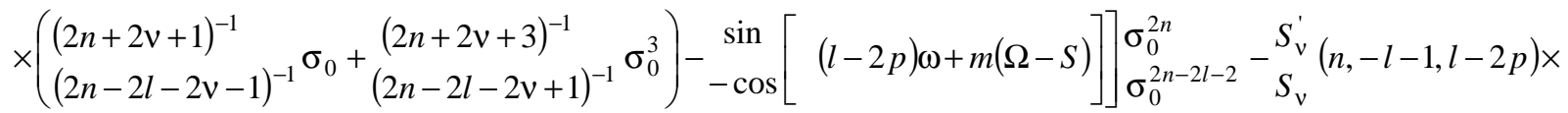

$$
\begin{aligned}
& \times\left[2 \sqrt{1-\gamma}(l-2 p) \cos _{-\sin }^{\sin }[(l-2 p) \omega+m(\Omega-S)]\left(\begin{array}{l}
(2 v+2)^{-1} \\
(2 n-2 l-2 v-2)^{-1}
\end{array} \sigma_{0}+\begin{array}{l}
(2 v+4)^{-1} \\
(2 n-2 l-2 v)^{-1}
\end{array} \sigma_{0}^{3}\right)+\right. \\
& \left.\left.+{ }_{\sin }^{\cos }[(l-2 p) \omega+m(\Omega-S)]\right] \begin{array}{c}
\sigma_{0} \\
\sigma_{0}^{2 n-2 l-3}
\end{array}\right\} \sigma_{0}^{ \pm 2 v} \mid \begin{array}{l}
\sigma_{0} \\
\bar{\sigma}_{0}
\end{array},
\end{aligned}
$$

$\frac{\partial \gamma}{\partial C_{l m}}=\sum_{p=0}^{l} \sum_{n=0}^{\infty}\left(\frac{a_{e}}{q}\right)^{l} \frac{\gamma^{n}}{(1-2 \gamma)} F_{l m p}(i) \sum_{v=0}^{\infty}\left\{\begin{array}{l}N_{\mathrm{v}}^{\prime} \\ N_{\mathrm{v}}\end{array}(n,-l-1, l-2 p)\left[2 \gamma \sqrt{1-\gamma}(l-2 p)_{\cos }^{-\sin }[(l-2 p) \omega+m(\Omega-S)] \times\right.\right.$ $\left.\times\left(\begin{array}{l}(2 n+2 v+1)^{-1} \\ (2 n-2 l-2 v-1)^{-1}\end{array} \sigma_{0}+\begin{array}{l}(2 n+2 v+3)^{-1} \\ (2 n-2 l-2 v+1)^{-1}\end{array} \sigma_{0}^{3}\right)-(1-\gamma){ }_{\sin }^{\cos }[(l-2 p) \omega+m(\Omega-S)]\right] \begin{aligned} & \sigma_{0}^{2 n} \\ & \sigma_{0}^{2 n-2 l-2}-S_{v}^{\prime}\end{aligned}(n,-l-1, l-2 p) \times$ $\times\left[2 \gamma \sqrt{1-\gamma}(l-2 p){ }_{\sin }^{\cos }[(l-2 p) \omega+m(\Omega-S)]\left(\begin{array}{l}(2 v+2)^{-1} \\ (2 n-2 l-2 v-2)^{-1}\end{array} \sigma_{0}+\begin{array}{l}(2 v+4)^{-1} \\ (2 n-2 l-2 v)^{-1}\end{array} \sigma_{0}^{3}\right)+\right.$ 


$$
\begin{aligned}
& \left.\left.+(1-\gamma)_{\cos }^{-\sin }[(l-2 p) \omega+m(\Omega-S)]\right] \begin{array}{l}
\sigma_{0} \\
\sigma_{0}^{2 n-2 l-3}
\end{array}\right\} \sigma_{0}^{ \pm 2 v} \mid \begin{array}{l}
\sigma_{0} \\
\bar{\sigma}_{0}
\end{array}, \\
& \frac{\partial \gamma}{\partial S_{l m}}=\sum_{p=0}^{l} \sum_{n=0}^{\infty}\left(\frac{a_{e}}{q}\right)^{l} \frac{\gamma^{n}}{(1-2 \gamma)} F_{l m p}(i) \sum_{v=0}^{\infty}\left\{\begin{array} { l } 
{ N _ { \mathrm { v } } ^ { \prime } } \\
{ N _ { \mathrm { v } } }
\end{array} ( n , - l - 1 , l - 2 p ) \left[2 \gamma \sqrt{1-\gamma}(l-2 p){ }_{\sin }^{\cos }[(l-2 p) \omega+m(\Omega-S)] \times\right.\right.
\end{aligned}
$$

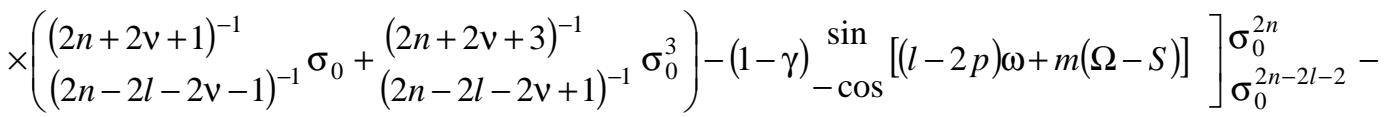

$$
\begin{aligned}
& -S_{S_{v}}^{S_{v}^{\prime}}(n,-l-1, l-2 p)\left[2 \gamma \sqrt { 1 - \gamma } ( l - 2 p ) { } _ { - \operatorname { c o s } } ^ { \operatorname { s i n } } [ ( l - 2 p ) \omega + m ( \Omega - S ) ] \left(\begin{array}{l}
(2 v+2)^{-1} \\
(2 n-2 l-2 v-2)^{-1}
\end{array} \sigma_{0}+\right.\right.
\end{aligned}
$$

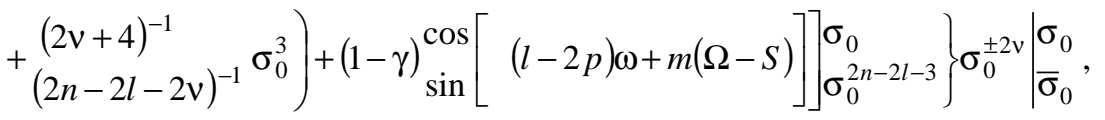

$$
\begin{aligned}
& \frac{\partial \sigma_{0}}{\partial C_{l m}}=\sum_{p=0}^{l} \sum_{n=0}^{\infty}\left(\frac{a_{e}}{q}\right)^{l} \frac{\gamma^{n}}{(1-2 \gamma)} F_{l m p}(i) \sum_{\mathrm{v}=0}^{\infty}\left\{\begin{array} { l } 
{ N _ { \mathrm { v } } ^ { \prime } } \\
{ N _ { \mathrm { v } } }
\end{array} ( n , - l - 1 , l - 2 p ) \left[\left(\frac{n(1-\gamma)}{\gamma}-l-1\right)_{\sin }^{\cos }[(l-2 p) \omega+m(\Omega-S)] \begin{array}{l}
(2 n+2 v+1)^{-1} \\
(2 n-2 l-2 v-1)^{-1}
\end{array}-\right.\right.
\end{aligned}
$$

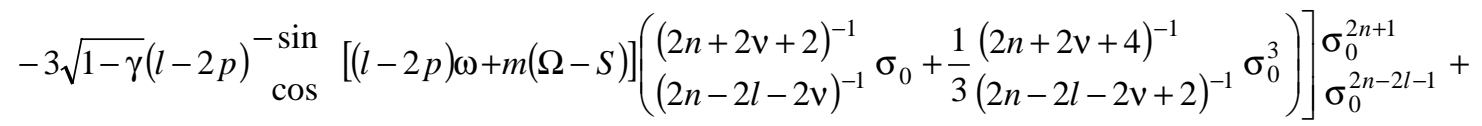

$$
\begin{aligned}
& +S_{S_{\mathrm{v}}}^{S_{\mathrm{v}}}(n,-l-1, l-2 p)\left[\left(\frac{n(1-\gamma)}{\gamma}-l-1\right)-\sin \left[((l-2 p) \omega+m(\Omega-S)] \begin{array}{l}
(2 v+2)^{-1} \\
(2 n-2 l-2 v-2)^{-1}
\end{array}+\right.\right. \\
& \left.\left.+3 \sqrt{1-\gamma}(l-2 p){ }_{\sin }^{\cos }[(l-2 p) \omega+m(\Omega-S)]\left(\begin{array}{l}
(2 v+3)^{-1} \\
(2 n-2 l-2 v-1)^{-1}
\end{array} \sigma_{0}+\frac{1}{3}(2 v+5)^{-1}(2 n-2 l-2 v+1)^{-1} \sigma_{0}^{3}\right)\right] \begin{array}{l}
\sigma_{0}^{2} \\
\sigma_{0}^{2 n-2 l-2}
\end{array}\right\} \sigma_{0}^{ \pm 2 v} \mid \begin{array}{l}
\sigma_{0} \\
\bar{\sigma}_{0}
\end{array}, \\
& \frac{\partial \sigma_{0}}{\partial S_{l m}}=\sum_{p=0}^{l} \sum_{n=0}^{\infty}\left(\frac{a_{e}}{q}\right)^{l} \frac{\gamma^{n}}{(1-2 \gamma)} F_{l m p}(i) \sum_{v=0}^{\infty}\left\{\begin{array} { l } 
{ N _ { v } ^ { \prime } } \\
{ N _ { v } }
\end{array} ( n , - l - 1 , l - 2 p ) \left[\left(\frac{n(1-\gamma)}{\gamma}-l-1\right){ }_{-\cos }^{\sin }[(l-2 p) \omega+m(\Omega-S)] \times\right.\right.
\end{aligned}
$$

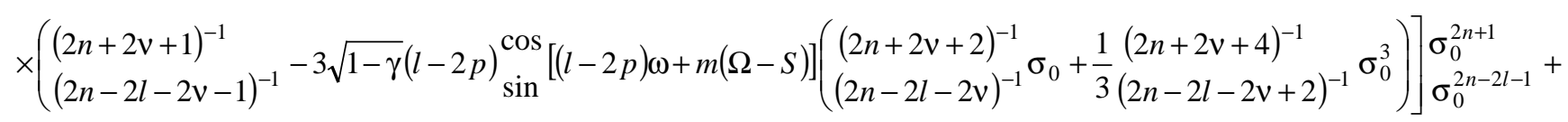

$$
\begin{aligned}
& +{ }_{S_{v}}^{S_{v}^{\prime}}(n,-l-1, l-2 p)\left[\left(\frac{n(1-\gamma)}{\gamma}-l-1\right)\right)_{\sin }^{\cos }[(l-2 p) \omega+m(\Omega-S)] \underset{(2 n-2 l-2 v-2)^{-1}}{(2 v+2)^{-1}}++3 \sqrt{1-\gamma}(l-2 p){ }_{-\cos }^{\sin } \times
\end{aligned}
$$

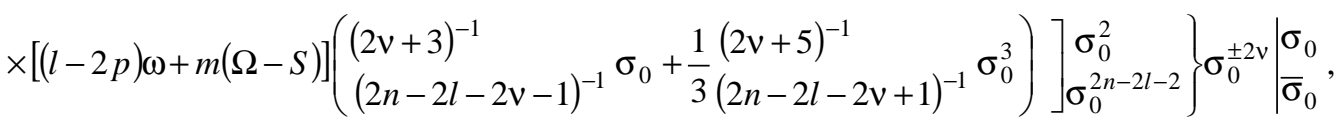

čia $a_{e}$ - vidutinis Žemès pusiaujo spindulys; $F_{l m p}(i)-$ palydovo orbitos posvyrio funkcija [15]; $S_{v}^{\prime}(n, \alpha, p), N_{v}^{\prime}(n, \alpha, p), S_{v}(n, \alpha, p), N_{v}(n, \alpha, p) \quad-$ skaitiniai koeficientai, priklausantys nuo indeksu $n, \alpha, p, v$ [13]. Formulèse (9-20) viršutinè eilutè atitinka atveji, kai $\sigma_{0}<1$, o apatine - kai $\sigma_{0}>1, \sigma_{0}$ laipsnio rodiklis teigiamas, kai $\sigma_{0}<1$, neigiamas - kai $\sigma_{0}>1$, viršutinès eilutès trigonometrinè funkcija imama tuo atveju, kai $l-m-$ lyginis skaičius, apatinès - kai $l-m-$ nelyginis skaičius. Formulèse yra trūkis, kai $\sigma_{0}=1$. Eilutes galima taikyti, kai $\left|\sigma_{0} \sqrt{\gamma}\right|<1$. Ženklas $\mid \begin{aligned} & \sigma_{0} \\ & \bar{\sigma}_{0}\end{aligned}$ reiškia, kad išvestinès prieaugis skaičiuotinas $\sigma_{0}$ kintant nuo $\bar{\sigma}_{0}$ iki $\sigma_{0}$.

Žemès gravitacinio parametro pataisos koeficientas nustatomas pagal formulę [4]

$$
\frac{\partial r^{\prime}}{\partial \mu}=\frac{\partial r^{\prime}}{\partial a} \frac{\partial a}{\partial \mu},
$$

čia $a$-palydovo orbitos didžioji pusašè,

$$
\frac{\partial r^{\prime}}{\partial a}=\frac{\partial r^{\prime}}{\partial x} \frac{\partial x}{\partial a}+\frac{\partial r^{\prime}}{\partial y} \frac{\partial y}{\partial a}+\frac{\partial r^{\prime}}{\partial z} \frac{\partial z}{\partial a} .
$$

Remdamiesi trečiuoju Keplerio dèsniu galime parašyti 


$$
\frac{\partial a}{\partial \mu}=\frac{a}{3 \mu}
$$

Taikydami nesutrikdytojo judejjimo formules gauname:

$$
\begin{aligned}
& \frac{\partial x}{\partial a}=(\cos u \cos \Omega-\sin u \sin \Omega \cos i) \frac{\left(1-e^{2}\right)}{1+e \cos v}, \\
& \frac{\partial y}{\partial a}=(\cos u \sin \Omega+\sin u \cos \Omega \cos i) \frac{\left(1-e^{2}\right)}{1+e \cos v}, \\
& \frac{\partial z}{\partial a}=\sin u \sin i \frac{\left(1-e^{2}\right)}{1+e \cos v}
\end{aligned}
$$

čia $u$ - platumos argumentas.

Pateiktosios kosminés geodezijos dinaminio metodo lygtys, tinkančios, kai DŽP judejjimas beveik parabolinis, gali būti taikomos punktų geocentrinėms koordinatėms, geopotencialo parametrams ir orbitos elementams patikslinti. Vietoje laiko taikomas naujas kintamasis $\sigma_{0}$, susijęs su parabolinio judejjimo tikraja anomalija. Lygčių koeficientai išreikšti dydžio $\gamma$ laipsninèmis eilutemis. Eilutès absoliučiai konverguoja esant ịvairioms palydovo orbitos ekscentriciteto reikšmėms nuo 0 iki 1 . Didèjant ekscentricitetui eilučiu konvergencijos greitis didèja, todèl jos ypač efektyvios, kai palydovo orbitos ekscentricitetas artimas vienetui.

Palydovų, judančių beveik parabolinėmis orbitomis, topocentrinems koordinatems nustatyti galima naudoti ivvairias šiuolaikines kosminès geodezijos priemones: lazerinius tolimačius, ilgos bazès radiointerferometrus ir kt. Tokių matavimų tikslumas ir remimasis pateiktaja teorija leidžia gauti naujos informacijos apie Žemès gravitacijos lauką, punktų geocentrines padètis bei beveik parabolini judejimą.

\section{Išvados}

Gautos dinaminio metodo lygtys, tinkančios palydovams, judantiems beveik parabolinėmis orbitomis, taikyti kosminės geodezijos uždaviniams spręsti. Lygčiu koeficientai išreikšti dydžio $\gamma$ laipsninėmis eilutėmis. Vietoje laiko taikomas naujas kintamasis $\sigma_{0}$, susijęs su parabolinio judejjimo, kurio perigejjaus nuotolis toks pats kaip ir palydovo judejimo, tikraja anomalija. Eilutès tinka, kai $\left|\sigma_{0} \sqrt{\gamma}\right|<1$. Ju konvergavimo greitis didejja, kai palydovo orbitos ekscentricitetas artejja prie vieneto. Dinaminio metodo lygtys gali būti taikomos geopotencialo parametrams, punktų geocentrinèms koordinatėms bei palydovo orbitos parametrams patikslinti.

\section{Literatūra}

1. Žalnierukas, A.; Petroškevičius, P. New Lithuanian Geodesy in turn of time (Neue Geodäsie in Litauen in der Zeit der letzten Umwandlungen). Schriftenreihe des DVW. Wiesbaden: Wittwer, 1998. Band 33, S. 71-81 (in German).

2. Petroškevičius, P.; Ramanauskas, R. Establishment of GPS fundamental network in Lithuania (Lietuvos valstybinio GPS tinklo sudarymas). Geodesy and Cartography (Geodezija ir kartografija), No 1 (21). Vilnius: Technika, 1995, p. 3-20 (in Lithuanian).

3. Zakarevičius, A. Coordinate systems of Lithuanian geodetic networks and their connection (Lietuvos geodezinių tinklų koordinačių sistemos ir jų ryšiai). Vilnius: Technika, 1996. 200 p. (in Lithuanian).

4. Baranov, V. N.; Boiko, J. G. and others. Satellite geodesy (Космическая геодезия). Moscow: Nedra, 1986. 407 p. (in Russian).

5. Klees, R.; Koop, R.; Visser, P. Et al. Efficient gravity field recovery from GOCE gravity gradient observations. Journal of geodesy, 74, No 7, 2000, p. 561-572.

6. Visser, P.; Van Den Ijssel, J.; Koop, R. et al. Exploring gravity field determination from orbit perturbations of the European Gravity Mission GOCE. Journal of geodesy, 75, No 2, 2001, p. 89-99.

7. Torge, W. The changing role of gravity reference networks. Geodesy on the Move. Gravity, Geoid, Geodynamics and Antarctica. IAG Scientific Assembly, Rio de Janeiro, Brasil, September 3-9, 1997. Springer, 1998, p. 1-10.

8. Sas-Uhrynowski, A.; Mroczek, S.; Sas, A.; Petroškevičius, P.; Obuchowski, R.; Rimkus, D. Establishment of Lithuanian national gravimetric first order network. Geodesy and Cartography (Geodezija ir kartografija), Vol XXVIII, No 3, Vilnius: Technika, 2002, p. 75-82.

9. Rapp, R. H. Past and future developments in geopotential modeling. Geodesy on the Move. Gravity, Geoid, Geodynamics and Antarctica. IAG Scientific Assembly, Rio de Janeiro, Brasil, September 3-9, 1997. Springer, 1998, p. 58-78.

10. Kern, M.; Schwarz, K.P.; Sneeuw, N. A study on the combination of satellite, airborne, and terrestrial gravity data. Journal of geodesy, 77, No 3-4, 2003, p. 217-225.

11. Plachov, J. V. Application of the theory of perturbations in the satellite geodesy (Применение теории возмущений в космической геодезии). Moscow: Nedra, 1983. 200 р. (in Russian).

12. Duboshin, G. N. Celestial Mechanics (Небесная механика). Moscow: Nauka, 1975. 799 p. (in Russian).

13. Petroškevičius, P. Dispersing in line the coordinations of nearly parabolic nonperturb movement (Разложение координат невозмущенного близпараболического движения в ряды). Papers of Geodesy (Geodezijos darbai), Vol IX, Vilnius, 1979, p. 121-132 (in Russian).

14. Petroškevičius, P. Equations of orbital method for nearparabolic movement (Orbitinio metodo lygtys, kai palydovo orbita artima parabolei). Geodesy and Cartography (Geodezija ir kartografija), Vol XXIX, No 3. Vilnius: Technika, 2003, p. 73-77 (in Lithuanian).

15. Petroškevičius, P. Nearly parabolic satellite movement in the Earth's gravity field (Движение близпараболического спутника в гравитационном поле Земли). Papers of Geodesy (Geodezijos darbai), Vol IX, Vilnius, 1979, p. 112-120 (in Russian). 